\title{
Effects of Common Thrombophilia Factor Mutations in Central Retinal Vein Occlusion
}

\author{
(D) Muhammer Ozgur Cevik, ${ }^{1}$ (1) Sadik Gorkem Cevik ${ }^{2}$ \\ 1Department of Medical Genetics, Adiyaman University Faculty of Medicine, Adiyaman, Turkey \\ ${ }^{2}$ Department of Ophthalmology, Bursa Yuksek Ihtısas Training and Research Hospital, Bursa, Turkey
}

\begin{abstract}
Objectives: Central retinal vein occlusion (CRVO) is a severe eye disease that impairs vision. Although numerous systemic conditions have been reported to be a contributor, its exact pathophysiology has not yet been resolved. The purpose of this study was to study the role of some common thrombophilic polymorphisms in CRVO patients.

Methods: A total of 33 CRVO patients ( 25 non-ischemic CRVO and 8 ischemic CRVO) and 30 controls were recruited. Factor $V$ Leiden (GI69IA), prothrombin (Factor II G202I0A), MTHFR (C677T), MTHFR (AI298C), and PAI-I 5G/4G polymorphisms in venous blood DNA samples were examined, as well as the presence of hypertension, diabetes mellitus, glaucoma, smoking, and history of thrombosis.

Results: It was determined that MTHFR C677T polymorphisms, either in heterozygous or homozygous form, might be a risk factor for CRVO and systemic thrombosis. No differences were detected between the CRVO and control groups in terms of diabetes mellitus $(p=0.058>0.05)$, hypertension $(p=0.3>0.05)$, smoking $(p=0.923>0.05)$, glaucoma $(p=0.06>0.05)$ or use of anticoagulant drugs $(p=0.4>0.05)$. Analysis of patient history revealed a statistically significant difference regarding a thrombotic event in the medical history of the CRVO group $(p=0.001<0.05 ; n=4)$ versus the control group. The ischemic CRVO group had a significantly higher incidence of diabetes mellitus $(p=0.002<0.05)$ and hypertension $(p=0.03 \mid<0.05)$ than the non-ischemic CRVO group.

Conclusion: The MTHFR C677T mutation appears to be a risk factor for CRVO but factor $V$ Leiden (GI69IA), prothrombin (Factor II G202I0A), MTHFR (AI298C), and PAI-I 5G/4G mutations were not determined to be specifically related to CRVO in this study. The presence of diabetes mellitus and hypertension was significant in the ischemic CRVO group. Further studies with larger sample sizes should be conducted.

Keywords: Central retinal vein occlusion; factor V Leiden (GI69IA); hereditary thrombophilia; MTHFR (AI298C); MTHFR (C677T); PAI-I 5G/4G; prothrombin (Factor II G202I0A).
\end{abstract}

\section{Introduction}

Retinal vein occlusion (RVO) is the clogging of veins that surround the retina. It is the second most common retinal vascular disorder after diabetic retinopathy. The outcomes of RVO can be severe, as it is one of the primary reasons for painless loss of vision or visual impairment (I). RVO can primarily be classified as branch retinal vein occlusion (BRVO) or central retinal vein occlusion (CRVO). Sometimes RVO may involve half of the fundus, which is known as hemiretinal vein occlusion. If obstruction of one of the dual trunks (i.e., involvement of only half of the retina surface) of the central retinal vein is observed, this anomaly is called hemicentral RVO, and obstruction of a significant branch of the central retinal vein at or near the optic disc is called hemispheric RVO (2). RVO is also classified as ischemic or non-ischemic occlusion, depending on the degree of non-perfusion based on a fluorescein angiogram (2). In the medical literature, sometimes cases of CRVO, BRVO, or even hemi-CRVO have 
been generalized under the name of RVO without considering the distinct characteristics of each variation. This is misleading, since there are substantial differences that affect the treatment approach $(2,3)$. For instance, BRVO is 4 to 6 times more prevalent than CRVO (4) since the worldwide prevalence of $\mathrm{RVO}$ is $0.052 \%$ for any RVO, $0.042 \%$ for BRVO, and $0.008 \%$ for CRVO (5). CRVO in young adults is uncommon; only $10 \%$ to $15 \%$ of patients with CRVO are under 40 years of age (6). Furthermore, the occlusion patterns of BRVO and CRVO are entirely dissimilar. In BRVO, a branch of the retinal vein system is occluded at the crossing of an artery and a vein. In CRVO, however, the occlusion is in the central vein that from a thrombus at the level of the lamina cribrosa, thus leading to obstruction of the total blood supply of the retina, causing retinal ischemia and edema plus decreased visual acuity. The pathological role of this point is not a coincidence: (a) as aging occurs, the lamina cribrosa's wall loses its elasticity, causing a compression of the vascular wall; (b) other degenerative processes, like those arising from the central retinal artery may cause compression on the contiguous venous wall; (c) the resulting turbulent flow disturbs the hemodynamics and of endoluminal thrombus formation (7).

The exact pathogenesis and ideal targeted treatments for CRVO remain unknown $(7,8)$. However, for all types of RVO, advanced patient age, chronic glaucoma, atherosclerotic risk factors (age, diabetes, and hypertension), hyperviscosity, coagulopathy, smoking, and migraine are predisposing conditions that may also be valid for CRVO $(7,8)$. CRVO can occur as a result of various pathological insults, including compression of the vein (mechanical pressure due to arteriosclerotic changes in the central retinal artery and/or structural changes in the lamina cribrosa), hemodynamic disturbances (associated with hyperdynamic or sluggish circulation), vasculitis (e.g., sarcoidosis), and changes in coagulating factors in the blood (e.g., deficiency of thrombolytic factors, increase in clotting factors). According to many researchers, CRVO can be considered a multifactorial pathological condition that corresponds to Virchow's triad (hemodynamic changes/venous stasis, degenerative changes of the vessel wall, and blood hypercoagulability) (8) with other contributing systemic factors (7). Among the elements of Virchow's triad, the contribution of thrombogenesis or hypercoagulability of blood has not been as well investigated as the other 2 two factors. Therefore, the contribution of various hematological abnormalities to the etiology, pathogenicity, and treatment of CRVO remains unclear and there are contrasting clinical results $(6-8,10)$.

Early recognition and treatment are essential to avoid potentially significant visual morbidity due to CRVO (II). Each contributing factor to CRVO pathology should be investigated. Given the conflicting findings and misgrouping of CRVO under RVO in most medical literature, the objective of this study was to perform a retrospective analysis of factor $V$ Leiden (GI69IA), prothrombin (Factor II) G202II0A, MTHFR (C677T), MTHFR (AI298C), and PAI-I 5G/4G mutations in CRVO patients and matched non-CRVO controls. To the best of our knowledge, this is the first study in the literature that focuses explicitly on CRVO and the contribution of hypercoagulative factors in a Turkish patient group.

\section{Methods}

This retrospective study was approved by the biomedical ethics committee of Yuksek Ihtisas Training Research Hospital, Bursa, Turkey. The study included patients examined between January 2015 and January 2017, and their data were retrospectively evaluated according to the principles of the Declaration of Helsinki. The patients were informed about treatment options and possible complications. Written, informed consent was obtained from the patients to use venous blood for genotyping and the collection of relevant health data in this research. The relevant data were restricted to details of hypertension, diabetes mellitus, glaucoma, smoking, and history of thrombosis.

Dilated retinal examination and fundus fluorescence angiography were performed. Fundus fluorescein angiography (FFA) and optical coherence tomography (OCT) findings were recorded for differentiation of ischemic and non-ischemic CRVO. A Topcon-900 device (Topcon GmbH, Berlin, Germany) was used for FFA and an Optovue device (Optovue Inc., Fremont, CA, USA) was to perform OCT. Ischemic CRVO was defined as a non-perfused area of $>10$ optic discs observed with FFA. Venous dilatation-tortuosity, retinal hemorrhage in all quadrants, and cotton wool spots were diagnosed as CRVO.

Participants' venous blood samples were genotyped for factor V Leiden, Factor II (G202IOA), MTHFR AI298C, MTHFR C677T, and PAI-I -675 4G/5G mutations. A factor $\checkmark$ Leiden kit (Roche Molecular Systems Inc., Pleasanton, CA, USA) was used for factor V Leiden, and a Factor II (prothrombin) G202IOA kit (Roche Molecular Systems Inc., Pleasanton, CA, USA) was used for prothrombin genotyping according to the manufacturer's instructions. A LightMix human factor $\mathrm{V}$ Leiden kit and a LightMix Factor II G202 I0A kit (TIB Molbiol $\mathrm{GmbH}$, Berlin, Germany) were used as positive controls. For MTHFR AI298C, MTHFR C677T, and PAI-I -675 4G/5G mutations, LightCycler FastStart DNA Master HybProbe (Roche Molecular Systems Inc., Pleasanton, CA, USA) was used with a LightMix Kit Factor XIII V34L kit (TIB Molbiol GmbH, Berlin, Germany) containing specific primers and probes for MTHFR AI298C, MTHFR C677T, and PAI-I -675 4G/5G. Reverse transcription polymerase chain reaction was monitored in real time. The gene polymorphism assessment was performed using a detailed melting curve analysis. 
The study data were analyzed using SPSS for Windows, Version I4.0 (SPSS Inc., Chicago, IL, USA) statistical software and Microsoft Excel (Microsoft Corp., Redmond, WA, USA) with a significance level of $p<0.05$.

\section{Results}

The study included 33 CRVO patients ( 14 male, 19 female) and 30 healthy controls ( 18 male, 12 female). All of the patients were diagnosed with CRVO (25 non-ischemic CRVO and 8 ischemic CRVO) with an average age of 48.21 years for the females (min-max: 23-74 years) and 51.86 years for the males (min-max: 35-78 years). The healthy control group had a similar age with no statistically significant difference: 47.8 years among the females (min-max: 23- 78 years) and 55.21 years among the males (min-max: $32-78$ years). In the CRVO group, 9 patients (30\%) had diabetes mellitus (ischemic CRVO: $n=5$, non-ischemic CRVO: $n=4$ ), 10 patients (33\%) had systemic hypertension (non-ischemic CRVO: $n=6$; ischemic CRVO: $n=4), 9$ patients $(30 \%)$ were cigarette smokers (non-ischemic CRVO: $n=7$; ischemic CRVO: $n=2$ ), 3 patients (10\%) had glaucoma (all non-ischemic CRVO), 3 patients $(10 \%)$ were using anticoagulants (all non-ischemic CRVO) (Table I).

Statistically, no differences were detected between the CRVO groups and the control group in terms of diabetes mellitus $(p=0.058>0.05)$, hypertension $(p=0.3>0.05)$, smoking $(p=0.923>0.05)$, glaucoma $(p=0.06>0.05)$, or usage of anticoagulant drugs $(p=0.4>0.05)$. The patient medical histories revealed a statistically significant difference in the presence of a thrombotic event in the CRVO group $(p=0.001<0.05 ; n=4)$ versus the control group. The ischemic CRVO group had a significantly higher rate of diabetes mellitus $(p=0.002<0.05)$ and hypertension $(p=0.03 \mathrm{I}<0.05)$ than the non-ischemic CRVO group.

The results of DNA analysis for hereditary mutations related to coagulation yielded a result of homozygous: $p=0.286<0.05$, heterozygous $p=0.123<0.05$ for factor $V$ Leiden; homozygous: no mutation observed in any participant, heterozygous: $p=0.137$ for Factor II G202 I0A; homozygous: no mutation observed in any participant, heterozygous: $p=0.15$ for MTHFR A I298C; and homozygous: $p=0.127$, heterozygous $p=0.125$ for PAI-I 4G/5G. No significant difference was observed between the CRVO and the control groups $(p=0.13 \mid>0.05)$. There was a statistically significant difference in the MTHFR C677T results for both homozygous $(p=0.01<0.05)$ and heterozygous $(p=0.01<0.05)$ mutations. No difference was observed between the non-ischemic and ischemic CRVO groups ( $p>0.05$ ).

\section{Discussion}

In this study, we did not find any correlation between CRVO and the factor $\mathrm{V}$ Leiden mutation. Factor $\mathrm{V}$ Leiden mutations cause Factor $V$ to be inactivated about 10 times slower than normal, which is associated with venous thrombosis (VT). The circulation slows, the generation of thrombin is increased and the result is a hypercoagulable state (thrombophilia) in either the heterozygous or the homozygous form. Activated protein $C$ is responsible for the inactivation of Factor Va and Factor VIIla in the coagulation cascade. In the presence of a mutant Factor $V$ (factor $V$ Leiden), this inhibition is interrupted, and blood clots form more rapidly (I2). However, these data are valid for thrombophilia in large

Table I. Patient characteristics

\begin{tabular}{|c|c|c|c|c|c|c|}
\hline \multirow[b]{3}{*}{ Participant Features } & \multicolumn{6}{|c|}{ Participant Classification } \\
\hline & \multicolumn{4}{|c|}{ CRVO } & \multicolumn{2}{|c|}{ NON-CRVO } \\
\hline & \multicolumn{2}{|c|}{ Male } & \multicolumn{2}{|c|}{ Female } & Male & Female \\
\hline Number of Participants & \multicolumn{2}{|c|}{14} & \multicolumn{2}{|c|}{19} & 18 & 12 \\
\hline Average Age (years) & \multicolumn{2}{|c|}{48.21} & \multicolumn{2}{|c|}{51.8} & 47.8 & 55.21 \\
\hline Ischemic CRVO & \multicolumn{2}{|c|}{5} & \multicolumn{2}{|c|}{3} & & \\
\hline \multirow[t]{2}{*}{ Non-Ischemic CRVO } & \multicolumn{2}{|c|}{18} & \multicolumn{2}{|c|}{7} & & \\
\hline & ICRVO & NCRVO & ICRVO & NCRVO & & \\
\hline Diabetes Mellitus & 3 & 3 & 2 & 1 & 6 & 4 \\
\hline Hypertension & 2 & 1 & 4 & 3 & 3 & 5 \\
\hline Cigarette Smoking & 5 & 1 & 2 & 1 & 6 & 3 \\
\hline Glaucoma & 0 & 2 & 0 & 1 & 2 & 1 \\
\hline
\end{tabular}

CRVO: central retinal vein occlusion; ICRVO: ischemic CRVO; NCRVO: non-ischemic CRVO. 
veins; the same may not be true for CRVO. We could not find any study specifically examining CRVO and a Factor $V$ Leiden mutation. Some researchers (I0) have found no statistically significant relationship between RVO and Factor $\mathrm{V}$ Leiden mutations. On the other hand, a meta-analysis reported a significant correlation between the Factor V GI69IA polymorphism and CRVO (I3).

Another thrombotic mutation, Factor II (prothrombin, G20210A), is the change of an amino acid from glycine to alanine at position 20210 of the 3'-untranslated region of the prothrombin protein gene. This change increases the plasma prothrombin level and raises the risk of VT 5 to 10 times by leading to enhanced 3'-end formation and increased mRNA and protein expression. Elevated prothrombin levels predispose to VT (14). We did not observe any difference between groups ( $p=0.137>0.05)$, and to our knowledge, no study in the medical databases has specifically analyzed CRVO and the prothrombin mutation.

Plasminogen activator inhibitor-I (PAI-I) suppresses the generation of active plasminogen, which eliminates fibrin from plasma (I5). The most-studied single-nucleotide polymorphism of the PAI-I gene is a single guanosine nucleotide insertion/deletion $(4 \mathrm{G} / 5 \mathrm{G})$ variation situated within the promoter region, 675 base pairs upstream of the transcription start site (16). The PAI-I 4G/5G mutation is reported to be associated with an increased risk of VT venous thrombosis, according to a recent meta-analysis (I7). To our knowledge, our study is the first examine a potential relationship to CRVO. We found no significant correlation; however, KuhliHattenbach et al. (I8) reported a positive correlation with retinal vein occlusion, without specifying the type.

A MTHFR C677T mutation, either in heterozygous or homozygous form, was determined to be a risk factor for CRVO in our study, which was consistent with research performed by Gao et al. (19) in a Chinese population. Although there is a meta-analysis report on the contribution of high levels of homocysteine in RVO pathology (20), the same consensus does not exist for MTHFR (C777T) mutations. Studies of different populations or ethnicities have had different results concerning (C677T) mutations (2I). Yioti et al. (22) from Greece and Ferrazzi et al. (23) from northern Italy demonstrated that the MTHFR C677T mutation was a risk factor for RVO.

Our results did not find a significant association with respect to MTHFR AI298C. In addition, we found no significant difference regarding cigarette smoking, diabetes, hypertension, and mean age between the CRVO and control groups. The primary limitation of our study, like many others in the literature, is the small sample size. Further studies of CRVO with larger population samples are needed.

In conclusion, we found that the MTHFR C677T muta- tion was a risk factor for CRVO. Factor $\mathrm{V}$ Leiden (GI69IA), prothrombin (Factor II G202IOA), MTHFR (AI298C), and PAI-I 5G/4G mutations were not determined to be specifically related to CRVO in this study.

\section{Disclosures}

Ethics Committee Approval: The ethics committee approval was obtained from biomedical ethics committee of Yuksek Ihtisas Training Research Hospital, Bursa, Turkey.

Peer-review: Externally peer-reviewed.

Conflict of Interest: None declared.

Authorship Contributions: Involved in design and conduct of the study (MOC); preparation and review of the study (MOC); data collection (SGC); and statistical analysis (SGC).

\section{References}

I. Sivaprasad S, Amoaku WM, Hykin P; RVO Guideline Group. The Royal College of Ophthalmologists Guidelines on retinal vein occlusions: executive summary. Eye (Lond) 2015;29:1633-8.

2. Hayreh SS. Prevalent misconceptions about acute retinal vascular occlusive disorders. Prog Retin Eye Res 2005;24:493-519.

3. Hayreh SS. Central Retinal Vein Occlusion. Ocular Vascular Occlusive Disorders. Cham: Springer; 2015. p. 621.

4. Rogers S, Mclntosh RL, Cheung N, Lim L, Wang JJ, Mitchell P, et al.; International Eye Disease Consortium. The prevalence of retinal vein occlusion: pooled data from population studies from the United States, Europe, Asia, and Australia. Ophthalmology 2010;1 I7:3 |3-9.el. [CrossRef]

5. Kolar P. Risk factors for central and branch retinal vein occlusion: a meta-analysis of published clinical data. J Ophthalmol 20।4;20I4:724780. [CrossRef]

6. Esmaili DD, Boyer DS. Recent advances in understanding and managing retinal vein occlusions [version I; referees: 3 approved]. FI000Research 2018;1-8. [CrossRef]

7. Marcucci R, Sofi F, Grifoni E, Sodi A, Prisco D. Retinal vein occlusions: a review for the internist. Intern Emerg Med 201 I;6:307-14. [CrossRef]

8. Keren S, Loewenstein A, Coscas G. Pathogenesis, prevention, diagnosis, and management of retinal vein occlusion. World J Ophthalmol 2014;4:92-I I2. [CrossRef]

9. Hayreh SS. Central Retinal Vein Occlusion. Ocular Vascular Occlusive Disorders. Cham: Springer; 2015. p. 637.

10. Russo PD, Damante G, Pasca S, Turello M, Barillari G. Thrombophilic mutations as risk factor for retinal vein occlu- sion: a case-control study. Clin Appl Thromb Hemost 2015;21:373-7.

II. Klein R, Moss SE, Meuer SM, Klein BE. The 15-year cumulative incidence of retinal vein occlusion: the Beaver Dam Eye Study. Arch Ophthalmol 2008;126:5/3-8. [CrossRef]

12. Rosendaal FR, Reitsma PH. Genetics of venous thrombosis. J Thromb Haemost 2009;7 Suppl I:30I-4. [CrossRef]

13. Zou Y, Zhang X, Zhang J, Ji X, Liu Y. Factor V GI69IA is associated with an increased risk of retinal vein occlusion: a meta- 
analysis. Oncotarget 2017;8:75467-77. [CrossRef]

14. Andreassi MG, Botto N, Maffei S. Factor V Leiden, prothrombin G20210A substitution and hormone therapy: indications for molecular screening. Clin Chem Lab Med 2006;44:5I4-2I.

15. Ulisse S, Baldini E, Sorrenti S, D'Armiento M. The urokinase plasminogen activator system: a target for anti-cancer therapy. Curr Cancer Drug Targets 2009;9:32-7I. [CrossRef]

16. Burzotta F, Di Castelnuovo A, Amore C, D'Orazio A, Di Bitondo $\mathrm{R}$, Donati $\mathrm{MB}$, et al. 4G/5G promoter PAl-I gene polymorphism is associated with plasmatic PAI-I activity in Italians: a model of gene-environment interaction. Thromb Haemost 1998;79:354-8.

17. Wang J, Wang C, Chen N, Shu C, Guo X, He Y, et al. Association between the plasminogen activator inhibitor-I 4G/5G polymorphism and risk of venous thromboembolism: a metaanalysis. Thromb Res 2014;134:I24I-8. [CrossRef]

18. Kuhli-Hattenbach C, Hellstern P, Nägler DK, Kohnen T, Hattenbach LO. Prothrombin polymorphism A 199IIG, factor V HR2 haplotype A4070G, and plasminogen activator-inhibitor-I polymorphism 4G/5G and the risk of retinal vein occlusion. Oph- thalmic Genet 2017;38:4I3-4I7. [CrossRef]

19. Gao W, Wang YS, Zhang P, Wang HY. MTHFR C677T mutation in central retinal vein occlusion: a case-control study in Chinese population. Thromb Res 2008; 121:699-703.

20. Li D, Zhou M, Peng X, Sun H. Homocysteine, methylenetetrahydrofolate reductase $\mathrm{C} 677 \mathrm{~T}$ polymorphism, and risk of retinal vein occlusion: an updated meta-analysis. BMC Ophthalmol 20।4; |4:147. [CrossRef]

21. Liew SC, Gupta ED. Methylenetetrahydrofolate reductase (MTHFR) C677T polymorphism: epidemiology, metabolism and the associated diseases. Eur J Med Genet 20I5;58:I-10.

22. Yioti GG, Panagiotou OA, Vartholomatos GA, Kolaitis NI, Pappa CN, Evangelou E, et al. Genetic polymorphisms associated with retinal vein occlusion: a Greek case-control study and meta-analysis. Ophthalmic Genet 2013;34:I30-9. [CrossRef]

23. Ferrazzi P, Di Micco P, Quaglia I, Rossi LS, Bellatorre AG, Gaspari G, et al. Homocysteine, MTHFR C677T gene polymorphism, folic acid and vitamin B 12 in patients with retinal vein occlusion. Thromb J 2005;3:13. [CrossRef] 